Article

\title{
Exact Solutions for Solitary Waves in a Bose-Einstein Condensate under the Action of a Four-Color Optical Lattice
}

\author{
Barun Halder ${ }^{1}$, Suranjana Ghosh ${ }^{2}$, Pradosh Basu ${ }^{1}$, Jayanta Bera ${ }^{1} \mathbb{D}$, Boris Malomed ${ }^{3,4, *(D)}$ and Utpal Roy ${ }^{1, *(\mathbb{D})}$ \\ 1 Department of Physics, Indian Institute of Technology Patna, Patna 801103, India; \\ barun.pph15@iitp.ac.in (B.H.); pradosh_2021ph23@iitp.ac.in (P.B.); jayanta.ppy14@iitp.ac.in (J.B.) \\ 2 Department of Physics, Indian Institute of Science Education and Research Kolkata, Kolkata 741246, India; \\ sghosh@iitp.ac.in or suranjana.ghosh15@gmail.com \\ 3 Department of Physical Electronics, School of Electrical Engineering, Faculty of Engineering, \\ Center for Light-Matter Interaction, Tel Aviv University, Ramat Aviv, Tel Aviv P.O. Box 39040, Israel \\ 4 Instituto de Alta Investigación, Universidad de Tarapacá, Casilla 7D, Arica 1000000, Chile \\ * Correspondence: malomed@tauex.tau.ac.il (B.M.); uroy@iitp.ac.in (U.R.)
}

Citation: Halder, B.; Ghosh, S.; Basu, P.; Bera, J.; Malomed, B.; Roy, U. Exact Solutions for Solitary Waves in a Bose-Einstein Condensate under the Action of a Four-Color Optical Lattice. Symmetry 2022, 14, 49. https:// doi.org/10.3390/sym14010049

Academic Editors: V.I. Yukalov, V. S. Bagnato and Rashid G. Nazmitdinov

Received: 27 November 2021

Accepted: 28 December 2021

Published: 31 December 2021

Publisher's Note: MDPI stays neutral with regard to jurisdictional claims in published maps and institutional affiliations.

Copyright: (c) 2021 by the authors. Licensee MDPI, Basel, Switzerland This article is an open access article distributed under the terms and conditions of the Creative Commons Attribution (CC BY) license (https:// creativecommons.org/licenses/by/ $4.0 /)$.

\begin{abstract}
We address dynamics of Bose-Einstein condensates (BECs) loaded into a one-dimensional four-color optical lattice (FOL) potential with commensurate wavelengths and tunable intensities. This configuration lends system-specific symmetry properties. The analysis identifies specific multiparameter forms of the FOL potential which admits exact solitary-wave solutions. This newly found class of potentials includes more particular species, such as frustrated double-well superlattices, and bichromatic and three-color lattices, which are subject to respective symmetry constraints. Our exact solutions provide options for controllable positioning of density maxima of the localized patterns, and tunable Anderson-like localization in the frustrated potential. A numerical analysis is performed to establish dynamical stability and structural stability of the obtained solutions, which makes them relevant for experimental realization. The newly found solutions offer applications to the design of schemes for quantum simulations and processing quantum information.
\end{abstract}

Keywords: four-color optical lattice; Bose-Einstein condensate; soliton

\section{Introduction}

A suitably prepared standing wave of laser radiation can form an optical lattice (OL), which are broadly used for trapping and steering ultracold atoms [1-8]. Offering a versatile platform for research in the area of matter waves, OLs have become the most appropriate candidate for the realization of quantum simulations [9-11]. Further, ultracold atoms and Bose-Einstein condensates (BECs) trapped in an OL are used as a basis for the development of atomic clocks, quantum sensors, quantum computers, and a variety of other applications in quantum technologies [12-14].

In particular, the study of BEC under the action of geometrically frustrated OLs has drawn much interest [15-18]. Many complex phenomena have been found in this connection, including Anderson-like localization and negative absolute temperature $[17,19-21]$. Optical superlattices subjected to frustration offer potential for the development of tools which can hold and mould robust matter-wave states, such as solitons [22-26]. Theoretical studies in this direction are chiefly limited to a variety of bi-color optical lattices (BOL). A more general form of multi-color OLs may offer additional advantages, including the following points: (i) the color (wavelength) and intensity of the constituent beams, building the effective optical potential, greatly influence the manner in which the atoms are trapped; (ii) the formation of solitons requires a specific correlation between the nonlinearity and the trap parameters, which the multi-color OL may help to maintain; (iii) relations between intensities of the constituent beams may be used to 
optimize the creation of the self-trapped patterns. Thus, multi-color beams can be used to design potentials necessary for holding complex soliton patterns.

The aim of this work is to introduce a four-color OL (FOL) with commensurate wavelengths, which acts on a cigar-shaped (quasi-one-dimensional) BEC with the cubic nonlinearity. The corresponding Gross-Pitaevskii equation (GPE) is used to find appropriate relations between the nonlinearity and the potential parameters which help to support solitons. We produce analytical solutions which identify the specific form of the FOL and its parameter domain which provide tunability of the soliton-building scheme. Many exact condensate wave functions are obtained, and the results are illustrated by several characteristic examples. These solutions may be used for applications similar to those proposed in previous works [27-32]. Stability of the exact wave functions is addressed by means of direct simulations, adding random perturbations either to the underlying stationary solution, or to the external trap (the latter implies the consideration of the structural stability of the exact solutions). We thus find that our solutions are fully stable, both dynamically and structurally.

\section{Exact Analytical Model for Obtaining the Solitary Excitations under the Novel FOL Trap}

The FOL potential is produced by the combinations of four OLs with commensurate wave numbers, $l, 2 l, 3 l$, and $4 l$, while the corresponding intensities of the laser beams, $V_{1,2,3,4}$, are treated as free parameters, with the intention to find appropriate relations between them. The corresponding effective potential acting on atoms is

$$
V(z)=\sum_{j=1}^{4} V_{j} \cos (j l z)
$$

The lattice depth may be compared to the recoil energy, $E_{R}=2 \pi^{2} \hbar^{2} /\left(M \lambda^{2}\right)$, and the scaled lattice wave-vector is given by $l=2 \pi a_{\perp} / \lambda$, where $\lambda$ is the wavelength, $M$ is the mass of the BEC atom, $a_{\perp}=\left(\hbar /\left(M \omega_{\perp}\right)\right)^{1 / 2}$ and $\omega_{\perp}$ is the transverse frequency. The dimensionless 1D-GPE has the form

$$
\left[i \frac{\partial}{\partial t}+\frac{1}{2} \frac{\partial^{2}}{\partial z^{2}}-g(z, t)|\psi(z, t)|^{2}-V(z)-i \tau(z, t)\right] \psi(z, t)=0 .
$$

Here, $g(z, t)$ is the nonlinearity coefficient, which may be made space- and timemodulated, while $\tau(z, t)$ represents the space- and time-modulated loss or gain of the condensate atoms. For illustration, we have exploited experimentally feasible parameters of $\mathrm{Li}^{7}$ BEC in the quasi-1D trapping configuration with transverse frequency $\omega_{\perp}=2 \pi \times 710 \mathrm{~Hz}$, OL wavelength $\lambda=10.62 \mu \mathrm{m}$, and scattering length $a_{s}=-0.21 \mathrm{~nm}$ corresponding to attractive interactions between atoms [33]. By varying the applied magnetic field and angle between the overlapping laser beams, it is possible to engineer the shape of the external potential [34,35].

To produce a spatially localized solution of Equation (2), following the general scheme used for engineering matter-wave configurations [36,37], we choose an ansatz,

$$
\psi(z, t)=A(z, t) F(B(z, t)) e^{i \theta(z, t)},
$$

such that the external potential is supposed to be found by precisely solving $B(z, t)$, amplitude $A(z, t)$, phase $\theta(z, t)$, and the condensate form factor $F[B(z, t)]$. We substitute this ansatz into the GPE (2) and separate out the real and imaginary parts. To establish relations between the physically relevant quantities like nonlinearity, amplitude, phase and external trap for a solitary wave solution, the real part can be mapped to the following nonlinear differential equation,

$$
\frac{\partial^{2} F[B(z, t)]}{\partial B(z, t)^{2}}-G F^{3}[B(z, t)]=0,
$$


which introduces a constant $G=2 g(z, t) A^{2}(z, t) / B_{z}^{2}(z, t)$ in the case of solitary wave excitations. $G$ is -1 for attractive and 1 for repulsive inter-atomic interactions. The last consistency condition in Equation (4) is nothing but the elliptic equation, whose exact solutions are well-known in the form of 12 Jacobian elliptic functions $(c n[z, m], s n[z, m]$, etc.), where $m$ is the modulus parameter with $0 \leq m \leq 1$ [38]. One can choose various shapes of the elliptic functions from periodic ( $m=0$ ) to localized $(m=1)$, by varying the value of its modulus parameter. Here, we will focus only on the localized forms of the elliptic functions, that is, $\operatorname{cn}[z, 1]=\operatorname{sech}[z]$, for the bright soliton case with attractive nonlinearity and $\operatorname{sn}[z, 1]=\tanh [z]$ for the dark soliton case with repulsive nonlinearity. In addition to solving the above-mentioned equation, we also obtain the following consistency relations:

$$
\begin{array}{r}
G B_{z}^{2}(z, t)-2 A^{2}(z, t) g(z, t)=0, \\
B_{t}(z, t)+B_{z}(z, t) \theta_{z}(z, t)=0,\left[A^{2}(z, t) B_{z}(z, t)\right]_{z}=0 \\
2 A(z, t) A_{t}(z, t)+\left[A^{2}(z, t) \theta_{z}(z, t)\right]_{z} \\
-2 \tau(z, t) A^{2}(z, t)=0 \\
\frac{A_{z z}(z, t)}{2 A(z, t)}-\frac{\theta_{z}^{2}(z, t)}{2}-\theta_{t}(z, t)-V(z)=0,
\end{array}
$$

where the subscripts stand, as usual, for partial derivatives. The above set of equations is solved simultaneously to produce

$$
\begin{aligned}
& B(z, t)=\frac{c(t)}{A^{2}(z, t)}, \quad \theta_{z}(z, t)=-\frac{A_{t}(z, t)}{A_{z}(z, t)}, \\
g(z, t)=G B_{z}^{2}(z, t) / 2 A^{2}(z, t), &
\end{aligned}
$$

where $c(t)$ is an arbitrary positive definite function of time. These equations indicate a direct dependence of phase and nonlinearity on the amplitude of the system which will be determined by the trapping potential through the last equation of the system (5).

We substitute the expression of the external potential from Equation (4) into the set of Equations (5) and (6) and obtain the amplitude, phase and nonlinearity in the following exact forms:

$$
\begin{aligned}
A(z, t) & =\sqrt{\frac{c(t)}{\gamma \exp \left(b_{1} \cos (l z)+b_{2} \cos (2 l z)\right)}}, \\
\theta(z, t) & =\frac{1}{16}\left(l^{2} b_{1}^{2}+l^{2} b_{2}^{2}\right) t, \tau(z, t)=\frac{1}{2} \frac{c^{\prime}(t)}{c(t)}, \\
g(z, t) & =\frac{G \gamma^{4}}{2 c^{2}(t)} \exp \left(4 b_{1} \cos (l z)+4 b_{2} \cos (2 l z)\right) .
\end{aligned}
$$

We here introduce two real constants, $b_{1}$ and $b_{2}$, which help us to define the final form of the FOL amplitudes:

$$
\begin{array}{cc}
V_{1}=\left(1+b_{2}\right) \frac{l^{2} b_{1}}{4}, & V_{2}=\left(\frac{-b_{1}^{2}}{16}+b_{2}\right) l^{2}, \\
V_{3}=-\frac{l^{2} b_{1} b_{2}}{4}, & V_{4}=-\frac{l^{2} b_{2}^{2}}{4} .
\end{array}
$$

This is one of the essential results of the present work. Constants $b_{1}$ and $b_{2}$ are thus identified as the prime tuning parameters for controlling the trapping potential and condensate density. For the attractive and repulsive interactions, assuming the commonly known bright- or dark-soliton solutions of the elliptic equation (Equation (4)), the condensate wave functions take, severally, the following form: 


$$
\begin{aligned}
& \psi(z, t)=\sqrt{\frac{c(t)}{\gamma \exp \left[b_{1} \cos (l z)+b_{2} \cos (2 l z)\right]}} \\
& \times \operatorname{sech}\left[\gamma \int_{0}^{z} \exp \left(b_{1} \cos (l z)+b_{2} \cos (2 l z)\right) d z^{\prime}\right] \exp (i \theta(z, t)), \\
& \psi(z, t)=\sqrt{\frac{c(t)}{\gamma \exp \left(b_{1} \cos (l z)+b_{2} \cos (2 l z)\right)}} \\
& \times \tanh \left[\gamma \int_{0}^{z} \exp \left(b_{1} \cos (l z)+b_{2} \cos (2 l z)\right) d z^{\prime}\right] \exp (i \theta(z, t)) .
\end{aligned}
$$

We are now in a position to analyze the relevant potential profiles and the corresponding condensate densities. Potential profiles are explained in Figures 1 and 2 . Condensate densities will be delineated in Figures 3 and 4 for some parameter domains of $b_{1}$ and $b_{2}$ with $c=0.1, g=0.1$, and $l=0.84$.

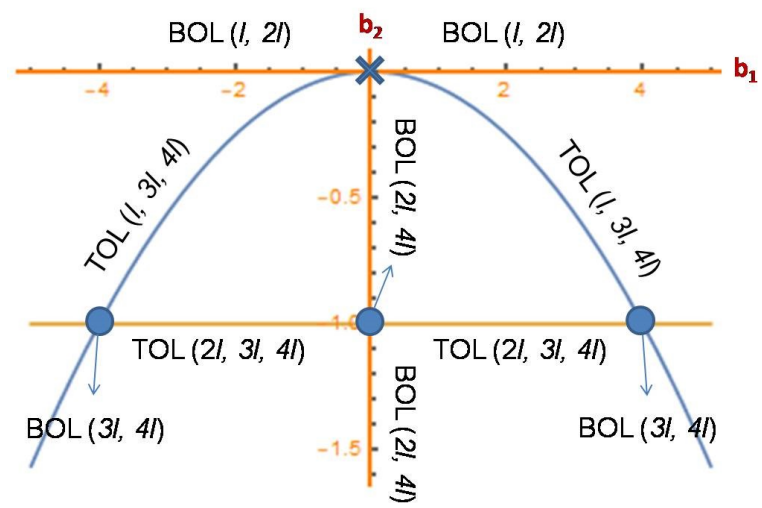

Figure 1. Curves and points for $l=0.84$ where the potential is not a FOL, but a TOL or a BOL. ' $\times$ ' signifies no potential for $b_{1}=b_{2}=0$. All other points in the $\left(b_{1}, b_{2}\right)$ plane correspond to FOLs.

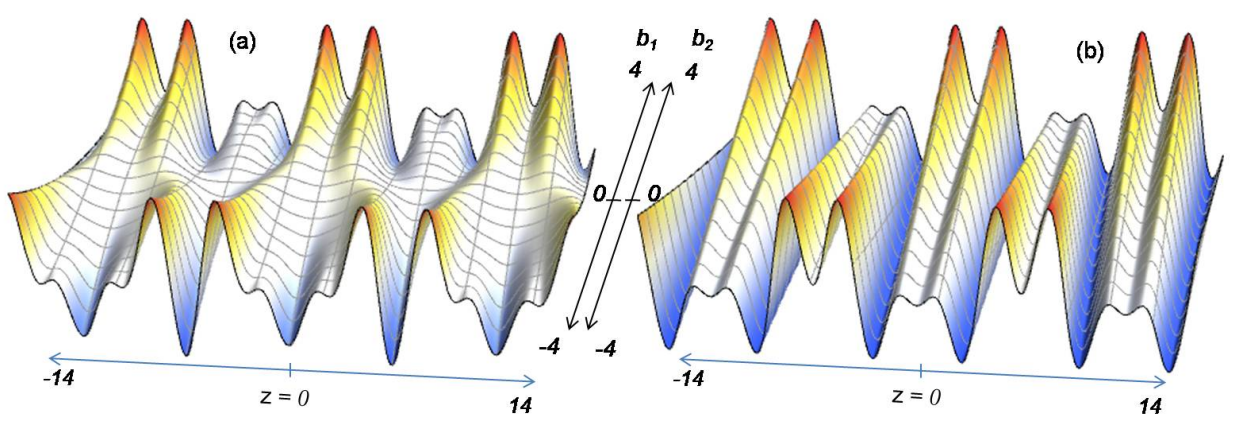

Figure 2. The variation of the FOL potential following the change of the tuning parameters for $l=0.84$ : (a) for fixed $b_{2}=2, b_{1}$ varies from -4 to $+4 ;$ (b) for fixed $b_{1}=2, b_{2}$ varies from -4 to +4 . Here and in the figures following below, the results are displayed in interval $-14<z<+14$. 

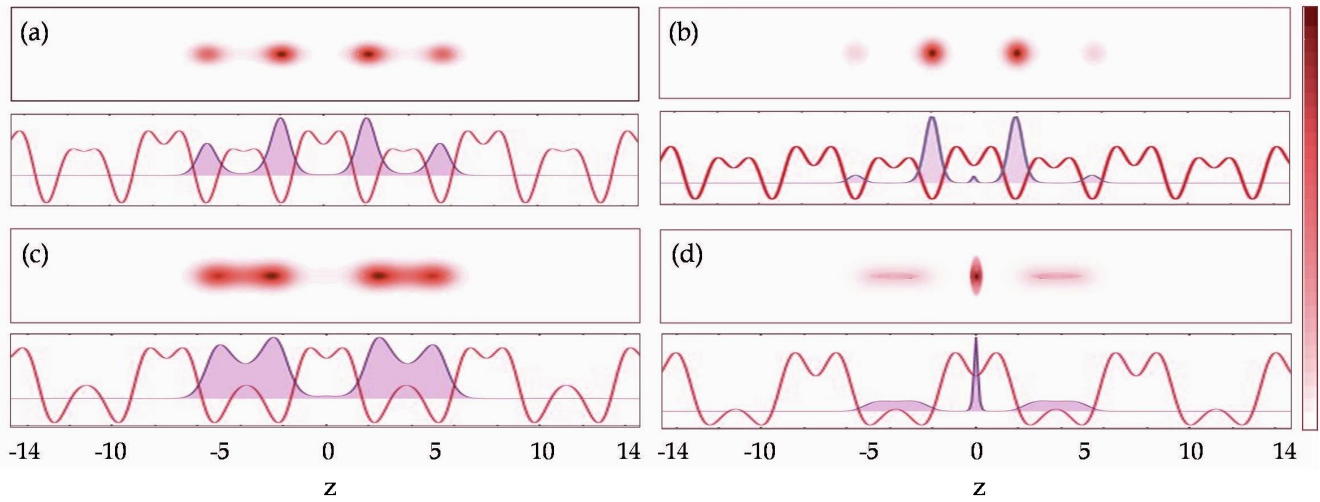

Figure 3. Condensate density patterns for $b_{1}>0$ and $b_{2}>0$ : (a) $b_{1}=1 ; b_{2}=2$, (b) $b_{1}=1 ; b_{2}=3.5$, (c) $b_{1}=2 ; b_{2}=1$, and (d) $b_{1}=3.5 ; b_{2}=1$. Each plot of (a-d) has two panels: the upper panel shows the contour plot of the density and the lower panel consists of a 2D plot of the density combined with the corresponding potential profile.
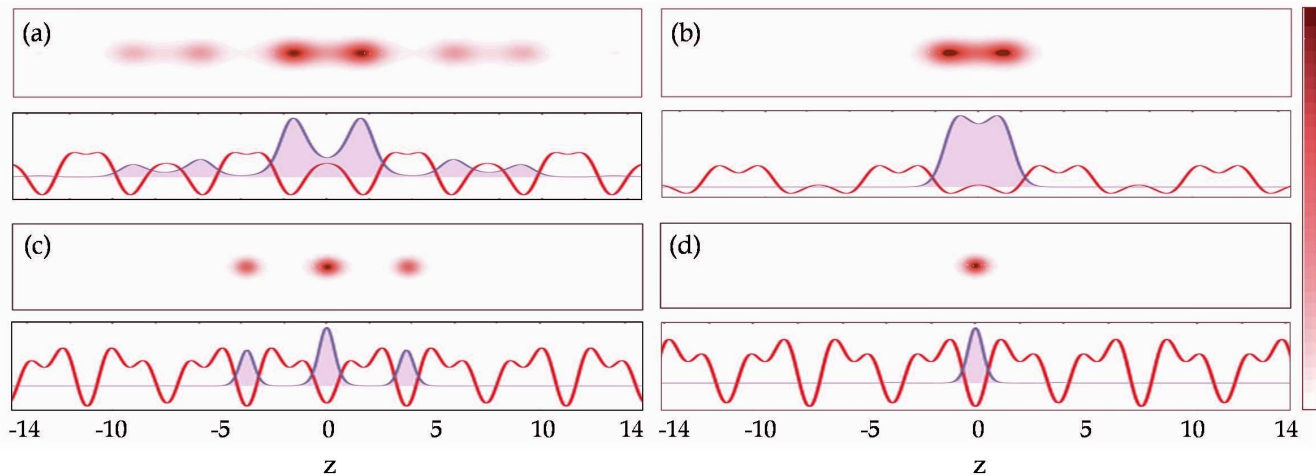

Figure 4. Condensate density patterns for $b_{1}<0$ or $b_{2}<0$ or both: (a) $b_{1}=-1 ; b_{2}=1$, (b) $b_{1}=-3$; $b_{2}=1,(\mathbf{c}) b_{1}=1 ; b_{2}=-3$, and (d) $b_{1}=-1 ; b_{2}=-3$. Each plot of (a-d) has two panels: the upper panel shows the contour plot of the density and the lower panel consists of a 2D plot of the density combined with the corresponding potential profile.

\section{The Parameter Domain and Shape of the Tunable FOL}

Figure 1 depicts the structure in the $\left(b_{1}, b_{2}\right)$ space, produced by Equation (9), where one obtains, as particular cases, a tri-color optical lattice (TOL), or a BOL. On the contrary, FOL is obtained in the entire space, excluding the curves and points indicated in the figure.

The respective FOL potential, drawn in Figure 2, seems interesting enough. For $b_{1}>0$ and $b_{2}>0$, the FOL is a disordered double-well superlattice, featuring frustrations in terms of both inter- and intra-well separations. Figure $2 \mathrm{a}, \mathrm{b}$ reduces to a $\mathrm{BOL}$ at $b_{1}=b_{2}=0$. However, the transition to the domain of $b_{1}<0$ or $b_{2}<0$ makes the potential shapes quite different. In the former case, a triple-well superlattice gradually appears at $b_{1}<0$, whereas in the latter case, a translational shift of the double-wells in the superlattice by half a period is observed. The presently engineered FOL may be the most advanced trapping potential for BEC, derived as an ingredient of exact solutions. It may find applications to the design of quantum simulation, information and computation schemes [28-31]. We will further illustrate the results by displaying density patterns.

\section{Density Patterns Supported by the Engineered FOL}

The density patterns in the domain of $b_{1,2}>0$ are displayed in Figure 3, along with the respective trapping profile, which help to understand the formation mechanism for the patterns. The presence of the inter- and intra-well potential frustration helps one to realize well-distinguished quantum clouds that may be employed for the design of enhanced atominterferometry (Figure $3 \mathrm{a}-\mathrm{c}$ ). When the intra-well frustration disappears, the previously 
separated clouds inside the double well become indistinguishable and the condensate starts accumulating at the central frustrated site, causing Anderson-like localization (Figure 3d). The wide tunability of the FOL and the corresponding mesoscopic clouds make it possible to predict a variety of quantum states that may be useful for quantum technology [39-41].

In Figure 4, we illustrate the situation in the negative domain: $b_{1}<0$ in Figure $4 \mathrm{a}, \mathrm{b}$, $b_{2}<0$ in Figure $4 \mathrm{c}$, and $b_{1,2}<0$ in Figure $4 \mathrm{~d}$. It produces several aligned, well-separated spatial Schrödinger-cat states for $b_{1}<0$ [42]. More negative $b_{1}$ offers localization of the cat-state at the central double well. For $b_{2}<0$, the resulting triple-well super-lattice generates an odd number of well-separated clouds. Interestingly, changing the sign of $b_{1}$ at $b_{2}<0$ spatially translates the triple-well lattice by one period to create a single BEC cloud at the center (Figure $4 \mathrm{~d}$ ). Thus, a transition from Figure $4 \mathrm{~d}$ to Figure $4 \mathrm{c}$ splits the single cloud into a set of three ones. In addition, a transition from Figure $4 \mathrm{~b}$ to Figure $4 \mathrm{a}$ splits the Schrödinger-cat state from one to three. Along with the above-mentioned possibilities, this scheme of potential engineering offers an efficient scheme for designing quantum logic gates $[31,32,43-46]$. To illustrate the temporal dynamics of one of the obtained solutions, we choose the trap corresponding to $b_{1}=2$ and $b_{2}=1$ which shows a frustrated doublewell super-lattice. Condensate, trapped in this potential, is allowed to evolve in time with a random noise of amplitude $10 \%$ of the maximum density. Condensate densities are depicted in Figure $5 \mathrm{a}-\mathrm{c}$ for $t=0, t=10 \mathrm{~ms}$, and $t=20 \mathrm{~ms}$, respectively. One can observe that the condensate is maintaining its shape after $t=10 \mathrm{~ms}$, but getting distorted at $t=20 \mathrm{~ms}$.

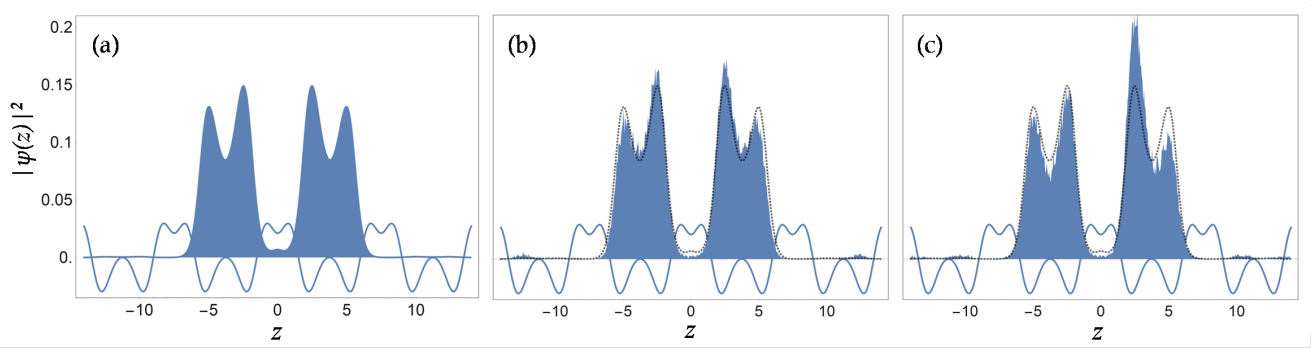

Figure 5. Condensate densities are depicted by filled plots at times (a) $t=0$, (b) $t=10 \mathrm{~ms}$, and (c) $t=20 \mathrm{~ms}$, along with the potential energy profile (solid-line curve) for $b_{1}=2$ and $b_{2}=1$. Initial density (dotted curve) is merged with the densities in $(\mathbf{b}, \mathbf{c})$ for reference.

\section{Dynamical Stability and Structural Stability of the Condensate}

It is obviously necessary to check the dynamical and structural stability of the special analytical solution produced above. The dynamical stability pertains to disturbance added to the wave function, while the structural stability implies deformation of the external trap. We addressed these problems separately by numerically solving the GPE with the help of the split-step Fourier method [21,24,32]. The results are presented in Figure 6. In the former case, we have added random white noise $\Re_{w}$ to the analytically obtained wave function, while in the latter case, the noise is added to the external trap. The noisy form of the initial wave function and potential are represented as

$$
\begin{aligned}
\psi_{\text {noisy }}(z, t & =0)=\psi(z, t=0)+\Re_{w} \\
V_{\text {noisy }}(z) & =V(z)+\Re_{w} .
\end{aligned}
$$

While the stability analysis was performed for a broad range of the parameters, here, we choose $b_{1}=2$ and $b_{2}=1$ for the purpose of illustration. In Figure 6, the condensate density profile, along with the external trap (not in scale), are depicted without the noise. The wave function is numerically evolved for both the noisy configurations defined as per Equation (10). Amplitude of noise $\Re_{w}$ varies from 0 to $5 \%$ of the maximum amplitude of the initial wave function. In the first scenario, we monitored the evolution of the wave functions, induced by the inputs $\psi_{\text {noisy }}(z, t=0)$ and $\psi(z, t=0)$, with our model potential, 
$V(z)$. To observe the stability of the stationary state, we computed deviation of the evolving condensate density $\left(D_{W}\right)$. In the latter case, we monitored the evolution of the input wave function $(\psi(z, t=0))$ under the action of the potentials $V_{\text {noisy }}(z)$ and $V(z)$, to observe the structural deformation in the condensate density $\left(D_{P}\right)$. We simulated the evolution for 10,000 time iterations with properly chosen space and time steps, $d z=0.277 \mu \mathrm{m}$ and $d t=0.22 \mu \mathrm{s}$, respectively. The deviation (maximum relative error) of the evolved noisy data from their noise-free counterparts is shown for both kinds of the stability analyses in Figure 6 by the upper curve $(*)$ and lower one, $\oplus$. Observing the noisy density profile after 10,000 iterations, we conclude that the density patterns retain their shapes with minimal deformation, which implies that the analytical solutions are indeed stable against both kinds of the random perturbations (Figure 6). The observed relative perturbation in the final configurations is near to $1 \%$ when the noise is initially added to the wave function, and near to $2 \%$ when it is added to the trapping potential. Thus, the presented model and its analytical solutions are physically relevant ones.

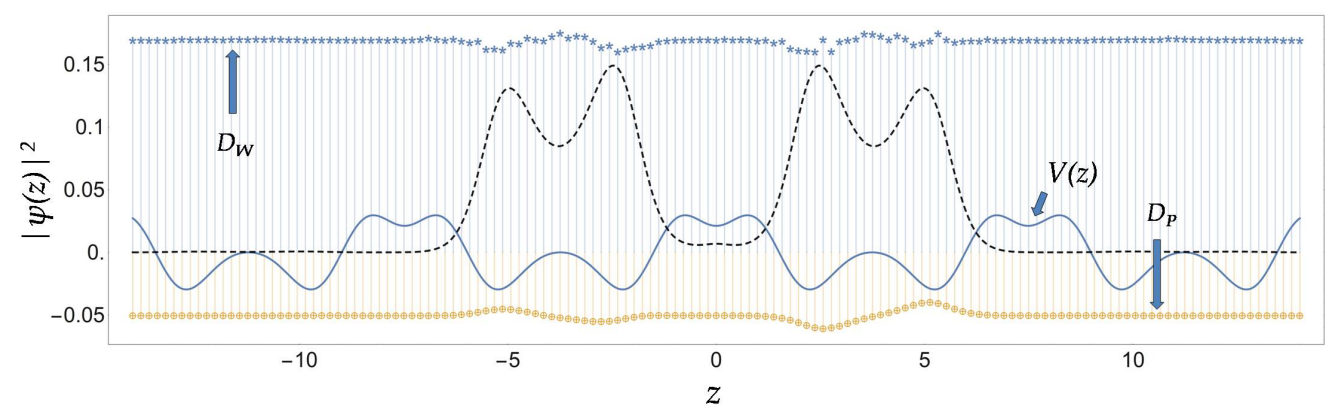

Figure 6. The numerical stability analysis of one of the obtained solutions with $b_{1}=2$ and $b_{2}=1$. The condensate density is depicted by the dotted line, and the trap profile, $V(z)$ (not in scale), is superimposed on it (the solid line). The deviation of the noisy data from their noise-free counterparts is shown for both kinds of the analyses: the dynamical stability, $D_{W}$ (the upper curve, composed of symbols $*$ ), and the structural stability, $D_{P}$ (the lower curve, composed of symbols $\oplus$ ).

\section{Conclusions}

In this paper, we reported the exact form of the four-color optical lattice (FOL) trap for the BEC in one dimension, which makes it possible to produce exact solutions for the trapped condensate. A variety of experimentally relevant trap profiles are reported, including one-, two-, three-, and four-color OLs with tunable shapes. It is worthy to stress that there are only two FOL-tuning parameters, $b_{1}$ and $b_{2}$, instead of four, making the detailed analysis of the exact solutions feasible. For chosen trap parameters, the exact condensate density is illustrated, and its variations after evolving in time are also shown. By means of systematic simulations, we have established dynamical and structural stability of the exact solutions. The stability against structural perturbations is especially important, as the solutions are valid only for the specially designed form of the FOL potentials. This class of FOL trapping potentials offers straightforward potential for use in applications, such as quantum simulation and other quantum technologies [13,28-31,39-41].

Author Contributions: Conceptualization, Data curation, Formal analysis, Investigation, Methodology, Software, Visualization, Validation; Writing—original draft, B.H. and S.G.; Data curation, Formal analysis, Investigation, Software-P.B. and J.B.; Writing-review and editing, B.M. and U.R.; Supervision, B.M. and U.R. All authors have read and agreed to the published version of the manuscript.

Funding: The work of B.A.M. was supported, in part, by the Israel Science Foundation through grant No. 1286/17.

Institutional Review Board Statement: Not applicable.

Informed Consent Statement: Not applicable. 
Conflicts of Interest: The authors declare no conflict of interest.

\section{References}

1. Bloch, I.; Dalibard, J.; Zwerger, W. Many-body physics with ultracold gases. Rev. Mod. Phys. 2008, 80, 885. [CrossRef]

2. Greiner, M.; Mandel, O.; Esslinger, T.; Hänsch, T.W.; Bloch, I. Quantum phase transition from a superfluid to a Mott insulator in a gas of ultracold atoms. Nature 2002, 415, 39-44. [CrossRef] [PubMed]

3. Denschlag, J.H.; Simsarian, J.E.; Häffner, H.; Mckenzie, C.; Browaeys, A.; Cho, D.; Helmerson, K.; Rolston, S.L.; Phillips, W.D. A Bose-Einstein condensate in an optical lattice. J. Phys. B At. Mol. Opt. Phys. 2002, 35, 3095. [CrossRef]

4. Jaksch, D.; Bruder, C.; Cirac, J.I.; Gardiner, C.W.; Zoller, P. Cold Bosonic Atoms in Optical Lattices. Phys. Rev. Lett. 1998, 81, 3108. [CrossRef]

5. Brazhnyi, V.A.; Konotop, V.V. Theory of nonlinear matter waves in optical lattices. Mod. Phys. Lett. B 2004, 18, 627-651. [CrossRef]

6. Morsch, O.; Oberthaler, M. Dynamics of Bose-Einstein condensates in optical lattices. Rev. Mod. Phys. 2006, 78, 179-212. [CrossRef]

7. Lewenstein, M.; Sanpera, A.; Ahufinger, V. Ultracold Atoms in Optical Lattices: Simulating Quantum Many-Body Systems; Oxford University Press: Oxford, UK, 2012.

8. Dutta, O.; Gajda, M.; Hauke, P.; Lewenstein, N.; Luhmann, D.S.; Malomed, B.; Sowinski, T.; Zakrzewski, J. Non-standard Hubbard models in optical lattices: A review. Rep. Prog. Phys. 2015, 78, 066001. [CrossRef] [PubMed]

9. Hauke, P.; Cucchietti, F.M.; Tagliacozzo, L.; Deutsch, I.; Lewenstein, M. Can one trust quantum simulators? Rep. Prog. Phys. 2012, 75, 082401. [CrossRef] [PubMed]

10. Gross, C.; Bloch, I. Quantum simulations with ultracold atoms in optical lattices. Science 2017, 357, 995-1001. [CrossRef]

11. Schäfer, F.; Fukuhara, T.; Sugawa, S.; Takasu, Y.; Takahashi, Y. Tools for quantum simulation with ultracold atoms in optical lattices. Nat. Rev. Phys. 2020, 2, 411-425. [CrossRef]

12. Brennen, G.K.; Pupillo, G.; Rey, A.M.; Clark, C.W.; Williams, C.J. Scalable register initialization for quantum computing in an optical lattice. J. Phys. B At. Mol. Opt. Phys. 2005, 38, 1687. [CrossRef]

13. Katori, H. Optical lattice clocks and quantum metrology. Nat. Photonics 2011, 5, 203-210. [CrossRef]

14. Wang, Z.M.; Wu, L.A.; Modugno, M.; Byrd, M.S.; Yu, T.; You, J.Q. Fault-tolerant breathing pattern in optical lattices as a dynamical quantum memory. Phys. Rev. A 2014, 89, 042326. [CrossRef]

15. Schulte, T.; Drenkelforth, S.; Kruse, J.; Ertmer, W.; Arlt, J.; Sacha, K.; Zakrzewski, J.; Lewenstein, M. Routes towards Anderson-Like Localization of Bose-Einstein Condensates in Disordered Optical Lattices. Phys. Rev. Lett. 2005, 95, 170411. [CrossRef] [PubMed]

16. Adhikari, S.K.; Salasnich, L. Localization of a Bose-Einstein condensate in a bichromatic optical lattice. Phys. Rev. A 2009, 80, 023606. [CrossRef]

17. Nath, A.; Roy, U. Bose-Einstein condensate in a bichromatic optical lattice: An exact analytical model. Laser Phys. Lett. 2014, 11, 115501. [CrossRef]

18. Yamamoto, D.; Fukuhara, T.; Danshita, I. Frustrated quantum magnetism with Bose gases in triangular optical lattices at negative absolute temperatures. Nat. Phys. 2020, 3, 56. [CrossRef]

19. Billy, J.; Josse, V.; Zuo, Z.; Bernard, A.; Hambrecht, B.; Lugan, P.; Clement, D.; Sanchez-Palencia, L.; Bouyer, P.; Aspect, A. Direct observation of Anderson localization of matter waves in a controlled disorder. Nature 2008, 453, 891-894. [CrossRef]

20. Braun, S.; Ronzheimer, J.P.; Schreiber, M.; Hodgman, S.S.; Rom, T.; Bloch, I.; Schneider, U. Negative Absolute Temperature for Motional Degrees of Freedom. Science 2013, 339, 52-55. [CrossRef]

21. Nath, A.; Bera, J.; Ghosh, S.; Roy, U. Exact Analytical Model for Bose-Einstein Condensate at Negative Temperature. Sci. Rep. 2020, 10, 9016. [CrossRef]

22. Cheng, Y.; Gong, R.; Li, H. Dynamics of two coupled Bose-Einstein Condensate solitons in an optical lattice. Opt. Exp. 2006, 14, 3594-3601. [CrossRef]

23. Das, P.; Raju, T.S.; Roy, U.; Panigrahi, P.K. Sinusoidal excitations in two-component Bose-Einstein condensates in a trap. Phys. Rev. A 2009, 79, 015601. [CrossRef]

24. Nath, A.; Bera, J.; Ghosh, S.; Panigrahi, P.K.; Roy, U. Soliton dynamics for an ingenious trap combination in a Bose-Einstein condensate. Eur. Phys. J. D 2020, 74, 27. [CrossRef]

25. Sun, Q.; Hu, J.; Wien, L.; Liu, W.M.; Juzeliunas, G.; Ji, A.C. Ground states of a Bose-Einstein Condensate in a one-dimensional laser-assisted optical lattice. Sci. Rep. 2016, 6, 37679. [CrossRef] [PubMed]

26. Li, J.; Zeng, J. Dark matter-wave gap solitons in dense ultracold atoms trapped by a one-dimensional optical lattice. Phys. Rev. A 2021, 103, 013320. [CrossRef]

27. Malomed, B.A. Soliton Management in Periodic Systems; Springer: Berlin/Heidelberg, Germany, 2006.

28. Qiu, X.; Zou, J.; Qi, X.; Li, X. Precise programmable quantum simulations with optical lattices. NPJ Quantum Inf. 2020, 6, 87. [CrossRef]

29. Windpassinger, P.; Sengstock, K. Engineering novel optical lattices. Rep. Prog. Phys. 2013, 76, 086401. [CrossRef]

30. Ghosh, S.; Bera, J.; Panigrahi, P.K.; Roy, U. Sub-fourier quantum metrology through bright solitary trains in Bose-Einstein condensate. Int. J. Quant. Inf. 2019, 17, 1950019. [CrossRef]

31. Yang, B.; Sun, H.; Huang, C.J.; Wang, H.Y.; Deng, Y.; Dai, H.N.; Yuan, Z.S.; Pan, J.W. Cooling and entangling ultracold atoms in optical lattices. Science 2020, 369, 550-553. [CrossRef] 
32. Bera, J.; Ghosh, S.; Salasnich, L.; Roy, U. Matter-wave fractional revivals in a ring waveguide. Phys. Rev. A 2020, 102, 063323. [CrossRef]

33. Khaykovich, L.; Schreck, F.; Ferrari, G.; Bourdel, T.; Cubizolles, J.; Carr, L.D.; Castin, Y.; Salomon, C. Formation of a Matter-Wave Bright Soliton. Science 2002, 296, 1290-1293. [CrossRef]

34. Inouye, S.; Andrews, M.R.; Stenger, J.; Miesner, H.J.; Stamper-Kurn, D.M.; Ketterle, W. Observation of Feshbach resonances in a Bose-Einstein condensate. Nat. Phys. 1998, 392, 151-154. [CrossRef]

35. Roberts, J.L.; Claussen, N.R.; Cornish, S.L.; Wieman, C.E. Magnetic Field Dependence of Ultracold Inelastic Collisions near a Feshbach Resonance. Phys. Rev. Lett. 2000, 85, 728. [CrossRef] [PubMed]

36. Kengne, E.; Liu, W.-M.; Malomed, B.A. Spatiotemporal engineering of matter-wave solitons in Bose-Einstein condensates. Phys. Rep. 2021, 899, 1-62 [CrossRef]

37. Nath, A.; Roy, U. A unified model for an external trap in a cigar-shaped Bose-Einstein condensate. J. Phys. A Math. Theor. 2014, 47, 415301. [CrossRef]

38. Abramowitz, M.; Stegun, I.A. Handbook of Mathematical Functions, 1st ed.; Dover: New York, NY, USA, 1964.

39. Howards, L.A.; Weinhold, T.J.; Shahandeh, F.; Combes, J.; Vanner, M.R.; White, A.G.; Ringbauer, M. Quantum Hypercube States. Phys. Rev. Lett. 2019, 123, 020402. [CrossRef]

40. Shukla, N.; Akhtar, N.; Sanders, B.C. Quantum tetrachotomous states: Superposition of four coherent states on a line in phase space. Phys. Rev. A 2019, 99, 063813. [CrossRef]

41. Shukla, N.; Nimmrichter, S.; Sanders, B.C. Squeezed comb states. Phys. Rev. A 2021, 103, 012408. [CrossRef]

42. Cirac, J.I.; Lewenstein, M.; Molmer, K.; Zoller, P. Quantum superposition states of Bose-Einstein condensates. Phys. Rev. A 1998, 57, 1208. [CrossRef]

43. Zeng, B.; Zhou, D.L.; Xu, Z.; Sun, C.P.; You, L. Encoding a logical qubit into physical qubits. Phys. Rev. A 2005, 71, 022309. [CrossRef]

44. Foot, C.J.; Shooter, M.D. Double well potentials and quantum gates. Am. J. Phys. 2011, 79, 762. [CrossRef]

45. Vo, C.; Riedl, S.; Baur, S.; Rempe, G.; Durr, S. Remote Entanglement between a Single Atom and a Bose-Einstein Condensate. Phys. Rev. Lett. 2012, 109, 263602. [CrossRef] [PubMed]

46. Gajdacz, M.; Opatrny, T.; Das, K.K. An atomtronics transistor for quantum gates. Phys. Lett. A 2014, 378, 1919. [CrossRef] 